\title{
Initial Attitude Impact on Error Propagation of Single-axis Rotation Inertial Navigation System
}

\author{
Wen Hua ${ }^{a}$ Zhili Zhang, Zhaofa Zhou, He Chen, ZiHao Xu \\ Xi'an High Technology Research Institute, Xi'an 710025, Shanxi China \\ abite1apple@163.com
}

Keywords: SINS, single-axis rotation, error propagation character, gyro constant drift

\begin{abstract}
The rotation modulation technology of Inertial Navigation System (INS) is an error auto-compensation technique. In order to study the effects of the initial attitude to the influence of error propagation character of single-axis rotation INS, the single-axis rotation INS error model was built, and error propagation equations of Strap down Inertial Navigation System(SINS) was deduced and obtained from which the function between equivalent constant error of inertial device, then analysis the modulation form of gyro constant drift in single-axis continuous rotation and those influences to systemic error, and design the simulation experiments in arbitrary initial attitude, which shows that the best rotation modulation result appeared in horizontal condition.
\end{abstract}

\section{Introduction}

The rotation modulation technology is one of the main directions of inertial technology ${ }^{[1-3]}$, whose essence is error auto-compensation technology ${ }^{[4-6]}$. Article [7] raised the systemic error of non-rotating SINS; Article [8] proposed the concept of gyro constant drift and applied multi-position alignment scheme; Article [9] analyses error propagation character of INS. At present, the premise of the analysis of error propagation character of single-axis rotation INS is $\boldsymbol{C}_{b}^{n}=\boldsymbol{I}$, but carrier coordinate system is generally different from navigation coordinate system in laboratories or other places, so it is necessary to discuss the body initial attitude $\boldsymbol{C}_{b}^{n}$ to the effects of error propagation character of single-axis rotation INS.

\section{Auto-Compensate Principle of Rotation Modulation}

\subsection{Systemic Error Propagation Equation Under Static Base}

Under the condition of static base, $V_{E}=V_{N}=V_{U}=0, \dot{V}_{E}=\dot{V}_{N}=\dot{V}_{U}=0, \boldsymbol{f}^{n}=-\boldsymbol{g}^{n}=\left[\begin{array}{lll}0 & 0 & g\end{array}\right]^{T} . \delta \boldsymbol{V}_{U}$ And $\delta h$ are not taken into account when analyze the systemic error characteristic Equations on account of altitude channel of the INS navigation calculating is divergence. According to attitude, velocity and location error equqtions systemic error equations under the condition of static base, when omitting latitude error $\delta \lambda$, write the error equations into matrix form is

$$
\left[\begin{array}{c}
\delta \dot{V}_{E} \\
\delta \dot{V}_{N} \\
\delta \dot{L} \\
\dot{\phi}_{E} \\
\dot{\phi}_{N} \\
\dot{\phi}_{U}
\end{array}\right]=\left[\begin{array}{cccccc}
0 & 2 \omega_{i e} \sin L & 0 & 0 & -g & 0 \\
-2 \omega_{i e} \sin L & 0 & 0 & g & 0 & 0 \\
0 & \frac{1}{R_{M}+h} & 0 & 0 & 0 & 0 \\
0 & -\frac{1}{R_{M}+h} & 0 & 0 & \omega_{i e} \sin L & -\omega_{i e} \cos L \\
\frac{1}{R_{N}+h} & 0 & -\omega_{i e} \sin L & -\omega_{i e} \sin L & 0 & 0 \\
\frac{\tan L}{R_{N}+h} & 0 & \omega_{i e} \cos L & \omega_{i e} \cos L & 0 & 0
\end{array}\right]+\left[\begin{array}{c}
\delta V_{E} \\
\delta V_{N} \\
\delta L \\
\phi_{E} \\
\phi_{N} \\
\phi_{U}
\end{array}\right]+\left[\begin{array}{c}
\delta f_{E} \\
\delta f_{N} \\
0 \\
\delta \omega_{E} \\
\delta \omega_{N} \\
\delta \omega_{U}
\end{array}\right]
$$

According to error equations, $\delta \lambda$ is not taken into account when establishing error equations on 
account of latitude error $\delta \lambda$ emerging in other systemic error equations.

In order to facilitate the analysis, the earth approximates for the ball and ignores the elevation $h$, where it is $R_{M}=R_{N}=R$. The Eq. (1) denotes as

$$
\dot{\boldsymbol{X}}=\boldsymbol{F} \boldsymbol{X}+\boldsymbol{u}
$$

The Eq. (1) is carried out Laplace transforming under the condition of static base, error propagation equations are obtained and written as

$$
\boldsymbol{X}(s)=(s \boldsymbol{I}-\boldsymbol{F})^{-1}[\boldsymbol{X}(0)+\boldsymbol{u}(s)]=\frac{\boldsymbol{N}(s)}{\Delta(s)}[\boldsymbol{X}(0)+\boldsymbol{u}(s)]
$$

Where $\boldsymbol{N}(s)$ is adjoint matrix of system characteristic matrix $(s \boldsymbol{I}-\boldsymbol{F}) ; \Delta(s)$ is system characteristic equations,

$$
\Delta(s)=|s \boldsymbol{I}-\boldsymbol{F}|=\left(s^{2}+\omega_{i e}^{2}\right)\left[\left(s^{2}+\omega_{s}^{2}\right)^{2}+4 s^{2} \omega_{i e}^{2} \sin ^{2} L\right]
$$

Where $\omega_{s}=\sqrt{g / R} \approx 1.24 \times 10^{-3} \mathrm{rad} / \mathrm{s}$ and $\omega_{i e}=7.292115 \times 10^{-5} \mathrm{rad} / \mathrm{s}$.

On account of $\omega_{s}^{2} \gg \omega_{i e}^{2}$, Eq. (4) approximately rewrites as

$$
\Delta(s)=\left(s^{2}+\omega_{i e}^{2}\right)\left[s^{2}+\left(\omega_{s}+\omega_{i e} \sin L\right)^{2}\right]\left[s^{2}+\left(\omega_{s}-\omega_{i e} \sin L\right)^{2}\right]
$$

The characteristic roots of Eq. (5) is

$$
s_{1,2}= \pm \mathrm{j} \omega_{i e}, \quad s_{3,4}= \pm \mathrm{j}\left(\omega_{s}+\omega_{i e} \sin L\right), \quad s_{5,6}= \pm \mathrm{j}\left(\omega_{s}-\omega_{i e} \sin L\right)
$$

Analytical solutions which are the first six state variables in Eq. (1) are obtained from which Eq. (3) is executed inversion Laplace transform. Longitude error $\delta \lambda$ is independently computed on account of which it doesn't existed in Eq. (1). From Eq. (1), error equation $\delta \dot{\lambda}$ is

$$
\delta \dot{\lambda}=\frac{\delta V_{E}}{R_{N}+h} \sec L \approx \frac{\delta V_{E}}{R} \sec L
$$

Applying Eq. (1) with Laplace transform, $s \delta \lambda(s)$ can be written as

$$
s \delta \lambda(s)=\frac{\sec L}{R} \delta V_{E}(s)+\delta \lambda(0)
$$

Substituting $\delta V_{E}(s)$ into Eq. (14) and inversion Laplace transform, we can get analytic solution of longitude error $\delta \lambda$.

Under the condition of ignoring the oscillation term in the analytic expression, reserving of values and accumulation, the function relationship between the equivalent error of inertial devices and the system error can be obtained:

$$
\left\{\begin{array}{c}
\delta V_{E}=-\cos L \delta \omega_{N}-\frac{R \sin 2 L}{2} \delta \omega_{U}, \delta V_{N}=-\frac{\omega_{s}^{2} \cos L}{\omega_{s}^{2}-\omega_{i e}^{2}} \delta \omega_{U} \\
\phi_{E}=-\frac{\delta f_{N}}{g}, \phi_{N}=\frac{\delta f_{E}}{g}, \phi_{U}=\frac{1}{\omega_{i e} \cos L} \delta \omega_{E}+\frac{\tan L}{g} \delta f_{E} \\
\delta L=\frac{\sin L}{\omega_{i e}} \delta \omega_{N}-\frac{\cos L}{\omega_{i e}} \delta \omega_{U}+\frac{\delta f_{N}}{g} \\
\delta \lambda=\frac{\tan L}{\omega_{i e}} \delta \omega_{E}+\frac{\delta f_{E}}{g \cos L}-t \cos L \delta \omega_{N}-t \sin L \delta \omega_{U}
\end{array}\right.
$$

\subsection{Auto-Compensate Principle of Gyro Constant Drift}

Modulation of gyro real error in navigation coordinate can be given as

$$
\delta \boldsymbol{\omega}_{i s}^{n}=\boldsymbol{C}_{s}^{n} \delta \boldsymbol{\omega}_{i s}^{s}=\boldsymbol{C}_{b}^{n} \boldsymbol{C}_{s}^{b}\left[\left(\left[\delta K_{g}\right]+[\delta G]\right) \boldsymbol{\omega}_{i s}^{s}+\boldsymbol{\varepsilon}^{s}\right]
$$

Where Eq.(2) is general representation of gyro error modulation in single-axis rotation INS.

Assuming body coordinate is initially the same with navigation coordinate and IMU coordinate, we get $\boldsymbol{C}_{b}^{n}=\boldsymbol{I}, \boldsymbol{C}_{s}^{b}\left(t_{0}\right)=\boldsymbol{I}$.IMU is relative to the body doing uniform rotation of unti-clockwise, so we can get $\alpha=\omega_{c} t$. Substituting $\boldsymbol{C}_{b}^{n}=\boldsymbol{I}, \boldsymbol{C}_{s}^{b}\left(t_{0}\right)=\boldsymbol{I}, \quad \alpha=\omega_{c} t$ into Eq. (10), and $\delta \boldsymbol{\omega}_{i s}^{n}$ is integrated in a complete rotation cycle, we can get 


$$
\int_{0}^{T} \delta \omega_{i s}^{n} \mathrm{~d} t=\int_{0}^{2 \pi / \omega_{c}}\left[\begin{array}{c}
\varepsilon_{x}^{s} \cos \omega_{c} t-\varepsilon_{y}^{s} \sin \omega_{c} t \\
\varepsilon_{x}^{s} \sin \omega_{c} t+\varepsilon_{y}^{s} \cos \omega_{c} t \\
\varepsilon_{z}^{s}
\end{array}\right] \mathrm{d} t=\left[\begin{array}{c}
0 \\
0 \\
\varepsilon_{z}^{s} T
\end{array}\right]
$$

From Eq. (11), we can know that the result of integrating $\varepsilon_{x}^{s}$ and $\varepsilon_{y}^{s}$ in a complete rotation cycle is zero and single-axis modulation around $z_{s}$ has no inhibitory.

For the real single-axis inertial navigation system, in order to provide true heading for IMU in the test and use of the process angle, whose purpose is analyzing the alignment or accuracy of navigation, IMU has the function of automatic zero return function, so that it is coincidence between the $s$ coordinate system and the $b$ coordinate system. At this point, the azimuth reference prism or high precision north seeking instrument can provide the true value of the heading angle for the IMU and the carrier. $\boldsymbol{C}_{s}^{b}\left(t_{0}\right)=\boldsymbol{I}$ Is generally the objective existence in the real system? However, for generally not deliberately completely overlap the carrier coordinates and navigation coordinate system either in the laboratory or field conditions, it is necessary to analyze the influence of the initial carrier attitude error self-compensation effects.

When the IMU rotates around the axis $z_{s}$ at the angular velocity $\omega_{c}$, Eq. (11) is integrated with the integral period, and substituting the attitude matrix is an orthogonal matrix, we can get

$$
\int_{0}^{T} \delta \omega_{i s}^{n} \mathrm{~d} t=\left[\begin{array}{c}
C_{13} \varepsilon_{z}^{s} T \\
C_{23} \varepsilon_{z}^{s} T \\
C_{33} \varepsilon_{z}^{s} T
\end{array}\right]=\left[\begin{array}{c}
(\sin \gamma \cos \psi-\cos \gamma \sin \psi \sin \theta) \varepsilon_{z}^{s} T \\
(-\sin \gamma \sin \psi-\cos \gamma \cos \psi \sin \theta) \varepsilon_{z}^{s} T \\
\cos \gamma \cos \theta \varepsilon_{z}^{s} T
\end{array}\right]
$$

From Eq. (12), we know that on the one hand, at the time of $\boldsymbol{C}_{b}^{n} \neq \boldsymbol{I}$, the single-axis continuous rotation does not produce the constant term and accumulation of the state variables because single-axis continuous rotation can still change the gyro drift in the vertical plane of the rotating shaft and the integral of the whole period is zero; on the other hand, the gyro drift in the direction of the rotation axis produces a projection in the horizontal plane of the navigation coordinate system, which is related to the specific numerical value of the attitude angle.

Particularly, when $\gamma=0$ and $\theta=0$, the projection in the horizontal plane is zero and the modulation effect is equivalent to $\boldsymbol{C}_{b}^{n}=\boldsymbol{I}$. However, the " $\gamma=0, \theta=0 "$ is sufficient conditions, rather than the necessary and sufficient conditions, which is equivalent to the modulation effects that appeared under the condition of $\boldsymbol{C}_{b}^{n}=\boldsymbol{I}$.

\section{Simulation Test and Analysis}

In order to directly reflect the self-compensation effect of the rotating modulation process, the simulation experiment is based on the single axis continuous rotation scheme. In particular, the simulation process uses the MATLAB symbolic mathematical toolbox, starting from the model (1) system error model, to carry out the Laplace transform and inverse transform of symbolic expression. Finally, to make the analytical expression of theoretical derived from Eq. (11) and MATLAB symbol operation each other, the parameters of Laplace transform are substituted into the formula to get the corresponding image.

\subsection{Error Propagation Simulation of Carrier System and Navigation System}

In $t_{0}$ time, the coordinate system of the carrier, the navigation coordinate system and the IMU coordinate system are the same, or that is $\boldsymbol{C}_{b}^{n}=\boldsymbol{I}, \boldsymbol{C}_{s}^{b}\left(t_{0}\right)=\boldsymbol{I}$. The latitude of the carrier is $30^{\circ} \mathrm{N}$. IMU with respect to the carrier rotates clockwise to speed $\alpha=\omega_{c} t$ uniform rotation, rotating angular velocity $5 \%$, simulation time is $60 h$, and Gyro drift is $\varepsilon_{x}^{s}=\varepsilon_{y}^{s}=\varepsilon_{z}^{s}=0.005 \% h$. 

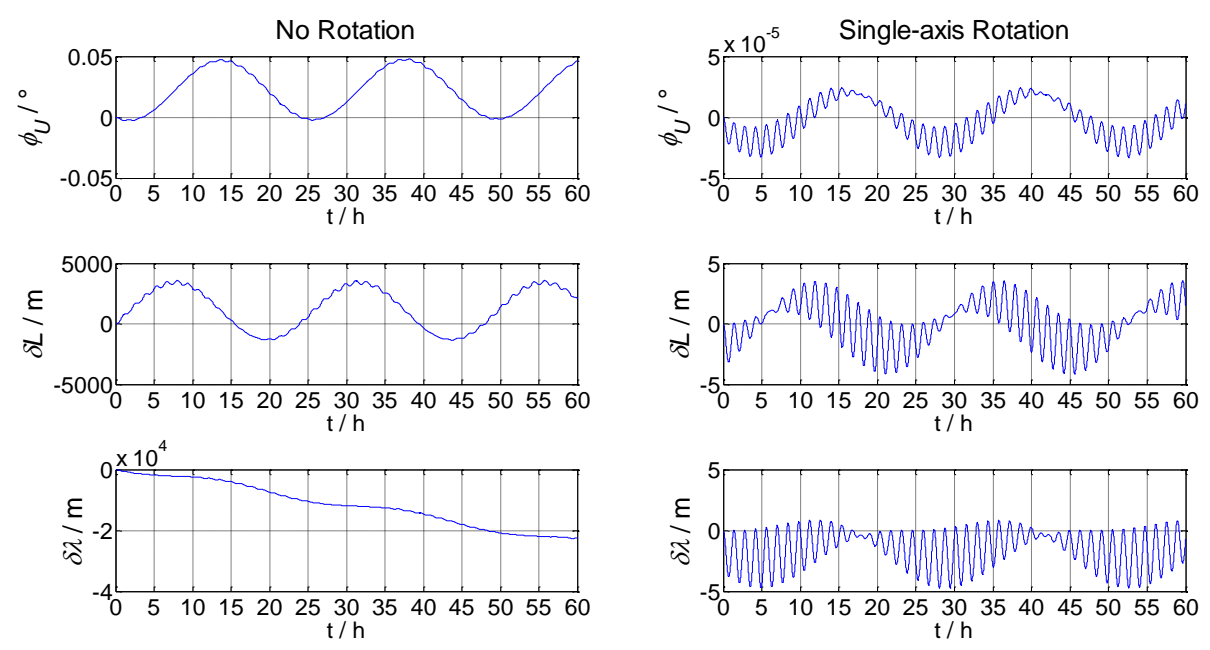

Figure $1 \mathrm{X}, \mathrm{Y}$ axis gyro drift caused by the navigation error contrast

As shown in figure 1, the constant error of the heading angle and latitude is caused by the gyro constant drift, and what's more, the error of the time accumulation is more serious besides error caused by the propagation of periodic oscillations without rotation modulation. The single axis rotation modulates the gyro constant drift into parameter of periodic variation the vertical plane of the rotation axis, of which the result is zero in the horizontal plane after integration, which makes that the navigation error of the constant term and accumulation item is obviously decreased with the constant drift of the horizontal gyro in the formula (11). The simulation results are in agreement with the theoretical analysis.

\subsection{Error Propagation Simulation of System and Navigation System in Any Attitude}
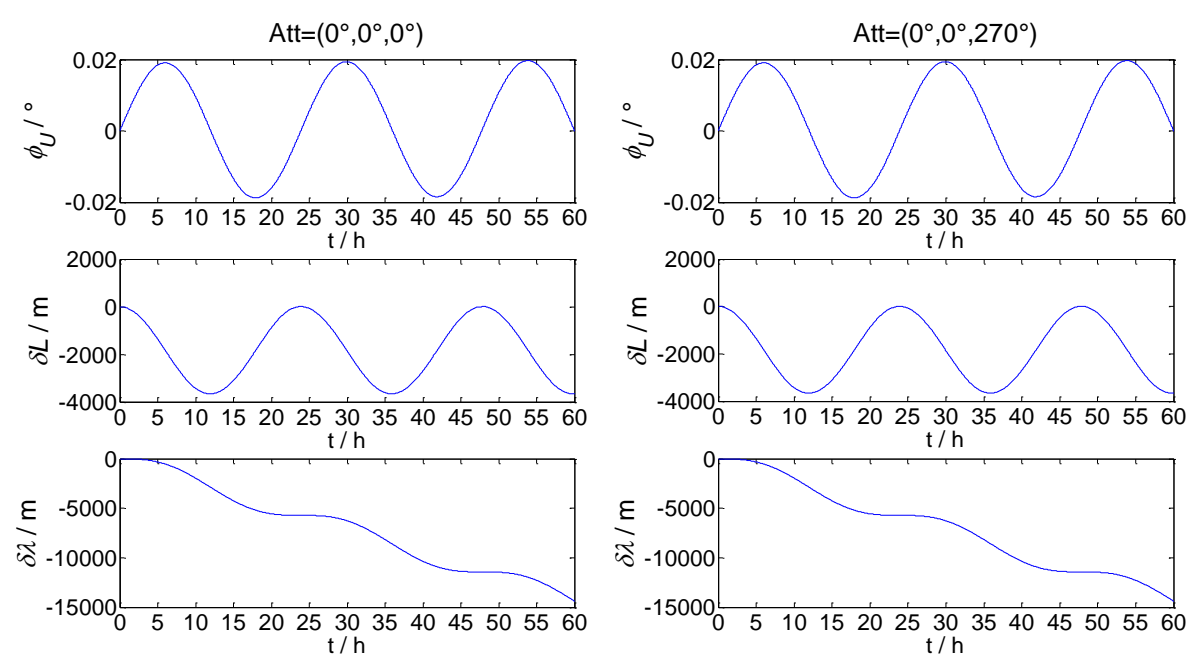

Figure 2 the navigation error of axis $\mathrm{Z}$ gyro drift caused by different initial attitude

As shown in figure 2, When the roll angle $\gamma=0^{\circ}$ and pitch angle $\theta=0^{\circ}$, it is consistent with the aforementioned theoretical analysis that the value of heading angle $\psi$ has no effect on the navigation error caused by gyro drift in axis $z_{s}$.

The main factors that affect the accuracy of heading angle are $\delta \omega_{E}$ according to Eq. (9). The vast majority of the initial alignment is $\psi \neq 0$ for actual single axis rotating inertial navigation system. For the specific $\psi$, although there are a variety of $\theta, \gamma$ combinations of making $\delta \omega_{E}=0$, other solutions of $\theta, \gamma$ can't be accurately obtained besides $(0,0)$ when the true value of $\psi$ is unknown or there is an error. In order to achieve the optimal rotation modulation effect and simplify the operation process, there is a level device to modulation carrier level that are $\theta=0, \gamma=0$. It should be as far as possible to choose a relatively flat terrain, if it is difficult to accurately leveling when vehicles carry out the initial on time out-door. 


\section{Conclusion}

In this paper, based on the error model of the SINS of rotational modulation, the error propagation equation of SINS is derived under the condition of static base, the function relation between the equivalent constant error of inertial device and the system error is obtained, the modulation form of gyro constant drift and its influence on the system error are analyzed when single-axis continuous rotates, The error propagation characteristics of gyro constant drift in the attitude of any carrier are simulated and the results are obtained: the rotation modulation effect is the best when the carrier is in horizontal state.

\section{References}

[1]. Ishibashi S, Tsukioka S, Sawa T, et al. The rotation control system to improve the accuracy of an inertial navigation system installed in an autonomous underwater vehicle. Workshop on Scientific Use of Submarine Cables and Related Technologies 2007.2007: 495-498.

[2]. Levinson Dr E, San Giovanni C Jr. Laser Gyro Potential for Long Endurance Marine Navigation. IEEE Position Location and Navigation Symposium, 1980: 115-129.

[3]. Terry T, Emanuel L. The AN/WSN-7B Marine Gyrocompass/Navigator. Proceedings of the 2000 National Technical Meeting of the Institute of Navigation. 2000: 348-357.

[4]. Huang W Q, Zhao G L, Tan Z F. Research of Gyro case rotating monitor technique [J]. Ship Building of China. 2002, 43: 54-59.

[5]. Yang Y, Miao L J, Shen J. Method of improving the navigation accuracy of SINS by continuous rotation [J]. Journal of Beijing Institute of Technology. 2005, 14(1): 45-49.

[6]. Hang Ling, Liu Jianye, Lai Jizhou. Rotating Fiber Optic Gyro Strapdown Inertial Navigation System with Three Rotating Axes [J]. Transactions of Nanjing University of Aeronautics \& Astronautics, 2008, 25(4): 289-29.

[7]. Sun Wei. Rotary Modulation Strapdown Inertial Navigation System [M]. Beijing: Surveying and Mapping Press, 2014

[8]. Xinlong Wang SINS Initial Alignment on Moving and Static Base [M] Xi'an: Northwest industrial University press, 2013.

[9]. SUN Feng, SUN Wei, Error Characteristic and Experiment Analysis of FOG SINS Based on Signal -axis Rotation [J]. Journal of Astronautics, 2010, 31(4):1070-1077. 